are to be warmly congratulated. One general feature, which was evident at last year's Fair and still more evident this year, was the increase in the number of scientific instruments used for industrial purposes, either for routine testing, for the,control of operations or in the field of scientific investigation. This tendency, it is safe to predict, will grow, and the extent to which it grows will form a fairly reliable indication of the extent to which scientific knowledge and scientific methods are permeating industry in general.

It is impossible, within the limits of an article, to do justice to all the points of interest in the combined exhibit of the scientific instrument manufacturers. Among the novel exhibits, C. Baker showed the 'Smith wear and lubricant tester'. This instrument has been designed and developed with the object of producing a reliable means of testing and investigating, by means of the same instrument, the hardness and wearing properties of various metals and also the lubricating and abrasive qualities of lubricants. Accordingly, the machine can be used to assist in the selection of the best lubricant for a given bearing or in the choice of bearing metal for a given purpose. The machine uses the principle common to certain other instruments, in which the revolving member wears a measurable impression in the flat surface of a test piece. It is, however, unique in many respects and particularly in that it employs as a wearing member a hardened steel ball of $1 \mathrm{~cm}$. diameter, exactly as is used in the Brinell hardness tester. Cooke, Troughton and Simms, Ltd., exhibited several new designs of surveying instruments, in which the size and weight have been considerably reduced without loss of accuracy, and the ease of reading the circles and the general facility for handling have been increased. Among these instruments, attention may be directed to the 'geodetic Tavistock theodolite', which reads to half a second of arc. In this instrument, the mean of the readings at both ends of a circle diameter is automatically indicated. The circle graduations are permanently marked on optical glass and both circles are read from a position near the eye-end of the telescope, whichever face is being read. This firm also showed a Vickers projection microscope, for which very great stability and rigidity are claimed. It is capable of carrying specimens up to $50 \mathrm{lb}$. in weight without any risk of damage.

Adam Hilger, Ltd., showed the 'Spekker photoelectric absorptiometer'. The principal advantage of this absorptiometer over the colorimeter of the Duboseq type is that visual matching is entirely eliminated. This firm also exhibited a new large spectrograph for metallurgical spectrum analysis. It is of the Littrow type and is unique in this respect, that it is the only type of spectrograph in the world in which all the adjustments are made automatically, simultaneously and accurately. One handle only is needed to effect these adjustments. W. Watson and Sons, Ltd., showed, among other exhibits, a profile projector and the Watson-Symon 'enlarging calculator'. The latter instrument is used in the production of enlargements of the highest standard from miniature negatives. It is claimed that the instrument can be used in combination with any type of enlarger and for any size of enlargement. $\mathbf{R}$. and $J$. Beck, Ltd., exhibited Dr. G. M. B. Dobson's 'ozone spectrometer'. It is claimed that by means of this instrument the thickness of the layer of ozone thirty miles above the earth can be measured and, from the measurement, a speedy and accurate weather forecast can be deduced. A comprehensive range of television receivers and equipment was shown on the stand of Baird Television, Ltd. The receivers incorporated a 'Cathovisor cathode ray tube', which is completely electro-magnetic in operation. A multiplier photo-electric cell was also shown and television lecture equipment for use in colleges and technical schools.

In view of the work on which the scientific instrument firms must be busily engaged in connexion with the great rearmament programme, it was no doubt difficult for many firms to exhibit at all and, for those firms who did exhibit, to give as comprehensive and detailed a picture of their industrial products as they would have liked. Nevertheless, as has been indicated, the scientific and optical instruments combined exhibit was a significant object lesson of the scope and vigour of this very vital industry.

\title{
Punjab Irrigation Research Institute*
}

$\mathrm{T}$ HE scope of the work undertaken by the Punjab Irrigation Research Institute, the director of which is Dr. E. McKenzie-Taylor, is illustrated in three publications, giving the results of different inquiries which have recently been pursued. In "The General Theory of the Gradient of Pressure under a Structure on Permeable Foundations" Mr. J. K. Malhotra and Dr. McKenzie-Taylor build up from first principles a general theory in conformity with the formulæ and values obtained by other workers for the rate of change of pressure in the direction of flow. Treating first the case of flow under a simple floor, a general expression for exit gradient is obtained, and this is extended to the case of flow under a complex structure, several specific cases being dealt with in order to show its applications.

* Punjab Irrigation Research Institute. Research Publications. Vol. 2, No. 16. Pp. 10. 3 annas, 4d. Vol. 3, No. 2. Pp. 25+iii + 4 plates. 10 annas, 18 . Vol. 3 , No. 3 . Pp. $47+10$ plates. 1.3 rupees, 18. 10d. (Lahore, 1937).
The second of these publications takes us from the structural interests of the Institute to that of cultivation. In the Punjab, it has been the custom to regard land suitable for rice as unfit for other crops, but the improvement noted during investigations on reclaimerl land tends to rebut this view. It is probable that land, which when first reclaimed is only fit for rice, can be improved to such an extent as to be capable of producing a normal rotation of crops. This is suggested in "The Soils of the Rice Areas of the Gujranwala and Sheikhupura Divisions of the Upper Chenab Canal" by Mr. M. L. Mehta.

In parts of the district referred to, the water table has risen considerably and, in consequence of the increased salt content and alkalinity, the land has gone out of cultivation. The objects of the investigation described were to determine the soil characteristics and the degree of deterioration of these lands as a first step towards their reclamation. 
From the numerous examinations of soil samples taken, certain areas where salt and alkalinity are high were indicated as being fit, as yet, only for rice crops. Others proved to be of better quality, and it is held that the failure to appreciate this is due to lack of knowledge of the proper method of rotation, the essential feature of which is to introduce a leguminous crop immediately after the rice in order to restore the nitrogen balance. The anrerobic soil conditions under which rice is grown have the result of reducing the nitrogen available for the succeeding crop. A study of the natural flora of the area showed that these gave reliable indications as to the state of the land.

This forms an interesting commentary on the third of these researches, which was suggested by the dominance of particular types of natural flora noticed in certain localities surveyed in 1931-32. The purpose of this investigation was to determine the class of soil fuvoured by each of the five types of vegetation selected, as a guide in future soil surveys. In "A Study of the Soil Profiles of the Punjab Plains with Reference to their Natural Flora" R. C. Hoon and M. L. Mehta deal fully with each case, and, in the result, show that four of the botanical types referred to-Prosopis spicigera, Salvadora oleoides, Tamarix articulata and Capparis aphylla-are re. liable indicators of the soils they favour, and deductions may be drawn as to the crops for which they are suitable and the precautions necessary when under irrigation and treatment. In conjunction with grass (Eleusine agyptica), however, a wide range of soils was found and from this type of vegetation, therefore, no index value was obtainable.

\section{The Aliphatic Diazo Compounds}

$I^{N}$ the forty-second Bedson Club Lecture delivered in King's College, Newcastle-upon-Tyne, Prof. J. Kenner discussed the aliphatic diazo compounds. The observation that the naturally occurring amide asparagine lost nitrogen when nitrous acid was present in nitric acid used for its hydrolysis led to the discovery of the general effect of this substance upon aliphatic amines. The work of Griess on aromatic diazo compounds was followed by the discovery of diazo acetic ester by Curtius, who turned to good account the suggestion of Baeyer that the acid part of glycine should be esterified whilst investigation of the amino group was proceeding. Together with Buchner, Curtius described the properties of the new substance which are responsible for the importance of its congeners to-day.

With regard to the formula of the aliphatic diazo compounds Curtius favoured the cyclic structure,

$$
\text { R.CH }
$$

but Thiele was led by observations of Staudinger and himself to propose $\mathrm{R} . \mathrm{CH}=\mathbf{N} \equiv \mathbf{N}$. Modern theory regards these compounds as examples of mesomerism, and the suggestion was now put for. ward that their formation from the diazo-hydroxides first produced, for example, in the preparation of diazomethane from nitrosomethylurethane, is due to the peculiar stability of the mesomeric state.

Prof. Kenner's active interest in the subject arose from his investigations of the tendency of the nitroso-group to pass whenever possible into the oximino-group. This led to an improvement of the early von Pechmann preparation of diazo paraffins, consisting in brief in the alkaline decomposition of the $\mathrm{N}$-nitroso derivative of the addition product of mesityl oxide and a primary aliphatic amine.

On the larger scale, certain difficulties were encountered. Loss was occasioned by the liberated diazoparaffin combining with the mesityl oxide simultaneously formed, but was reduced by working at lower temperatures and by separating mesityl oxide from the distillate by a fractionating column. In the case of higher homologues the boiling points of which approximate to that of mesityl oxide, pulegone can with advantage be substituted for the latter, in the preparation of the nitroso compound. Ether, first used to carry away the diazo compounds, was recognized by Meerwein to inhibit their reactivity, but a very satisfactory technique was developed in which anisole is used as solvent and nitrogen as a carrier gas under reduced pressure.

Vinyldiazomethane and its methyl derivative exhibit a comparatively feeble reactivity towards acids ; this was attributed to internal saturation, and in agreement with this the vinyl compound readily passes by monomolecular reaction into pyrazole.

After a discussion of some of the properties of the aliphatic diazo compounds, their chemical behaviour was discussed and more particularly towards ketones. The inertia of these towards diazomethane was first overcome when Meerwein activated acetone by means of methyl alcohol. The initial attack of the diazo compound at the carbon atom of the carbonyl group is followed by loss of nitrogen and stabilization, either by formation of an ethylene oxide ring or by a Wagner-Meerwein type of rearrangement. This latter, in the particular case of cyclic ketones, results in ring enlargement; cyclo-hexanone, for example, gives on treatment with diazomethane, 46 per cent cyclo-heptanone. Similar reactions have been carried out with the homologues of diazomethane (which are more reactive than the parent substance), and numerous 6-, 7- and 8-membered 2-alkyl cyclo. ketones have thus been prepared. The same products were prepared directly from the corresponding nitrosourethanes by a procedure applied by Meerwein to nitrosomethylurethane, and this has the advan. tage of applicability in cases in which it may not be possible to prepare the diazo compound itself. By this device also it is possible to introduce groups into a ketone which may be of use in further synthetic operations. 